\title{
ABC of Healthy Travel
}

\section{DIABETICS, CHILDREN, AND PREGNANT WOMEN}

\section{Diabetes mellitus}
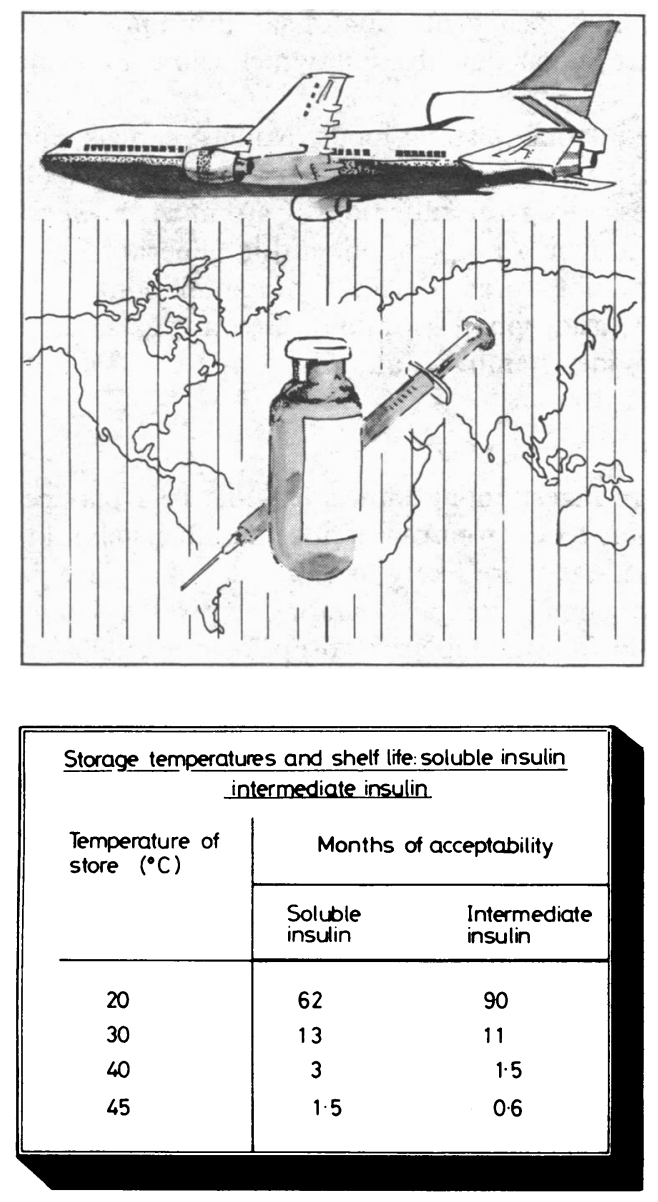

\section{Travelling with children}

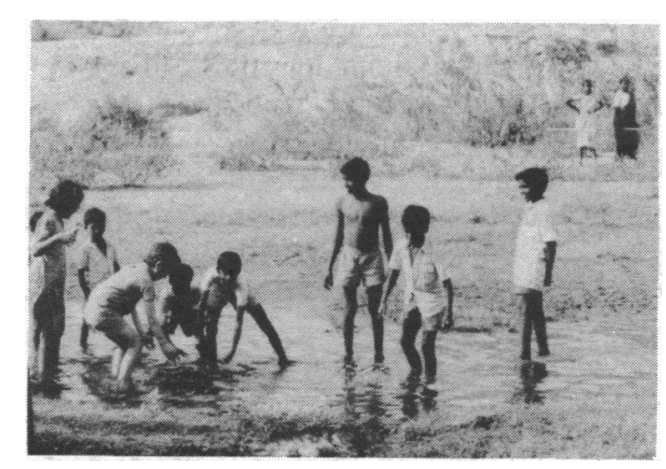

For short trips such as package holiday tours diet can be selected from normal menus. Emergency carbohydrate (sweets and starch) must be always available. Ample supplies of personal drugs, insulin, and testing equipment should be taken since new supplies obtained abroad may be initially difficult to identify because of different names, tablet sizes, and strengths.

Patients taking oral hypoglycaemic agents need not to change their schedules even when crossing time zones.

Patients on insulin should have individual schedules worked out by their doctor. The following are general guidelines.

When crossing many time zones it is convenient for the diabetic to remain on home time for injections and carbohydrate intake until arrival. After that each insulin injection can be altered by two to three hours until they are fully adjusted to the new local time. If the time between injections is longer than usual a small supplementary injection of soluble insulin may be needed if urine tests become very sugary. If the time between injections is shorter than usual a small temporary decrease in dose may be advisable, perhaps 4 or 8 units. Often it will take a few days to re-establish normal control and the need for urine tests every four to six hours over this period must be emphasised.

Disposable syringes are unbreakable and safe to reuse without cleaning with spirit even in the tropics. A few should be kept in hand luggage. Cooling of insulin is not essential for short trips (see table). Insulin should never be carried in luggage that is put in the hold of an aircraft, since it must not be frozen. Insulin obtained abroad may be of different type and strength. In the USA, Canada, New Zealand, and Australia insulin of $100 \mathrm{U} / \mathrm{ml}$ strength has replaced that of $80 \mathrm{U} / \mathrm{ml}$, and different syringes are needed. This change is now taking place in Britain.

Insulin must be continued during diarrhoeal or other illnesses, and the patient should also take easily absorbable carbohydrate. If vomiting occurs injections should continue and hospital care be sought. Ketoacidosis is rare in some races living in the tropics so the experience of local doctors may be limited. The accustomed facilities for managing eye, renal and neuropathic complications may also be unavailable. Companions should be aware of the condition. Advice on medical insurance is available in the British Diabetic Association's pamphlet on travel.

The generalisation that children travel well refers principally to their ability to enjoy new experiences. It must not be taken to mean that immunisations, preparation for travel, and other measures to prevent illness are less important than for adults. Children should be as fit as possible before departure, and this includes paying special attention to existing ailments and teeth. During air flights a contented child can prevent exhaustion of the parents, and if forewarned, most airlines can make special seating arrangements and provide cots for babies. They may also supply disposable nappies and special feeds, but it is wise to confirm this. At times a mild sedative such as chloral hydrate or an antihistamine during long flights is useful. 

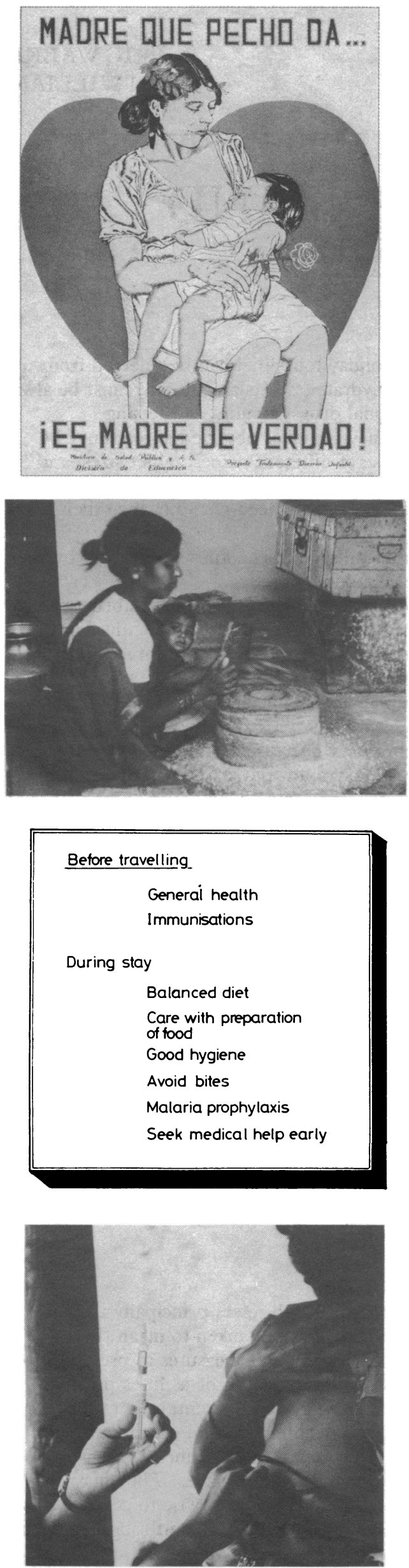

In many tropical countries child morbidity and mortality is much higher than in Britain. In general this does not apply to expatriates' children when they have adequate food, take care with hygiene, have received immunisations, and receive medical help early in illness. Medical facilities and the availability of drugs may, however, be wrongly taken for granted.

\section{Diet}

It is wise to breast feed infants and it may be convenient to continue this for longer than is usual in Britain-even well into the second year of life. Feeding babies with fresh or reconstituted dried cows' milk provides them with opportunities for ingesting pathogenic organisms through the milk or from utensils; also the higher concentration of salt in unmodified cows' milk contributes to hyperosmolar dehydration, which can complicate gastroenteritis.

Local advice may be obtained about weaning foods. Millets, cereals, and pulses can all be ground and used. Although convenient, tins of baby foods may be expensive and not necessarily more nutritious. Care must, however, be taken to avoid contamination during preparation, and in general feeds should be used immediately and not stored. Bacterial contamination develops more rapidly when the atmosphere is humid. Green vegetables and fruits are important to provide vitamins $\mathrm{A}$ and $\mathrm{C}$.

Teeth

When children are unlikely to ingest enough fluoride-that is, 1 part per million in water-to protect their teeth from caries fluoride tablets should be considered. Additional fluoride is more likely to be needed if surface water is drunk than if the water comes from artesian or deep wells. The amounts of fluoride contained in fluoride tablets or drops as prescribed in Britain are unlikely to do harm even when natural fluoride is present in the water, except in very young children in whom too much fluoride can cause mottling of the teeth. In an increasing number of countries it is now possible to find out the fluoride concentrations of public water supplies.

\section{Climate}

The long term effects of climate on children are difficult to assess because many other influences such as social, economic, dietary, and infective factors are involved. Although practices like placing a baby in a pram in the tropical sun must be avoided, a hot climate itself seems to do little harm to healthy children from temperate areas. Nevertheless, maintaining salt balance, avoiding sunburn, and wearing appropriate clothing are important. Prickly heat is more a problem in children than in adults.

\section{Immunisation and malarial prophylaxis}

When children are overseas it is wise to review immunisation procedures in the light of the practices of the host country. Diphtheria, pertussis, tetanus, and polio vaccinations are often begun as early as the first or second months of life where these diseases are widespread. Measles vaccine can be given at 9 months and again at about 15 months of age in regions where measles, because of its high prevalence, tends to infect children as soon as their maternally acquired immunity has waned. If given at birth BCG can prevent miliary or meningitic forms of tuberculosis in infants. For reasons not altogether clear severe malaria is unusual in babies during the first few months of life, but expatriates should give malarial prophylaxis to their children from birth. 


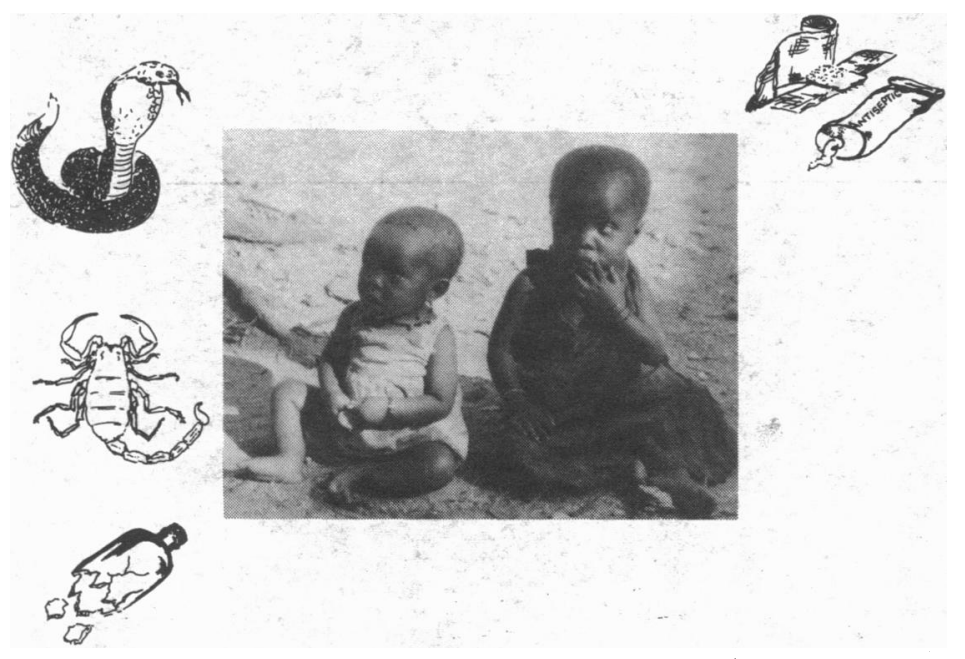

\section{Accidents and bites}

As children begin to crawl and walk they become more vulnerable to faecal-oral infections and hazards such as bites, accidents, and burns. Open wounds should be kept clean and covered with dressings until healed.

Deaths from scorpion bites are unusual but mostly occur in children aged under 2 years. Allowing toddlers to play outside unattended can be particularly hazardous.

Taking adequate malarial prophylaxis should not encourage the traveller to ignore the risks from other mosquito borne diseases such as dengue, which can be more severe in children. Protection from mosquito bites is also important in those children who are strongly allergic to them. Appropriate clothes and bed or window netting at night are usually more valuable in the long term than insect repellents.

\section{Pregnancy}
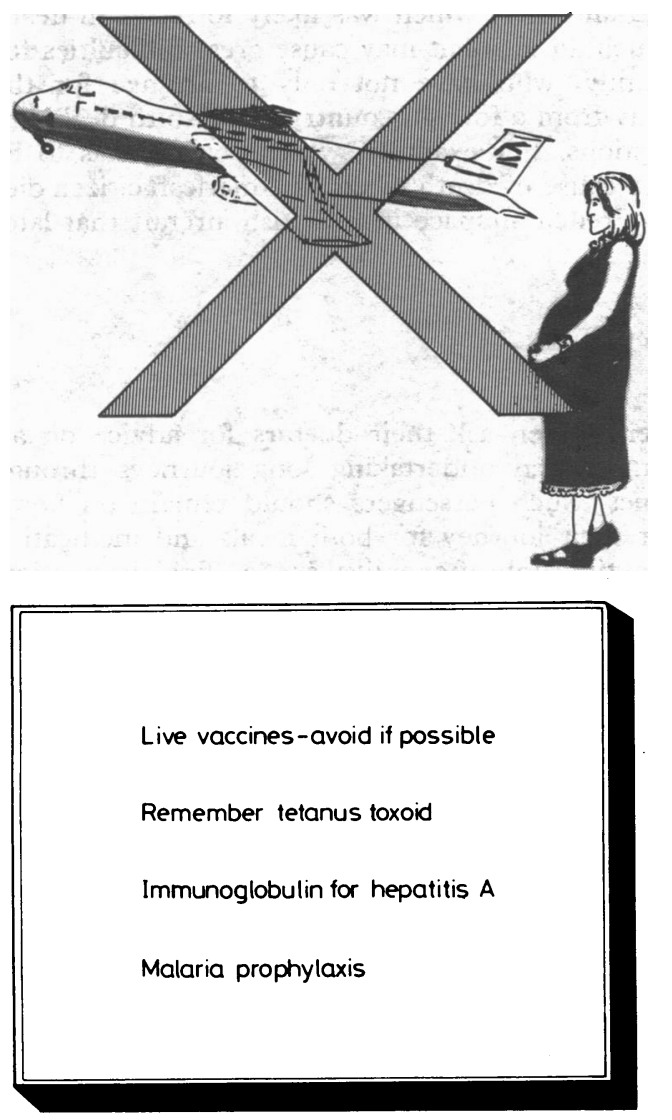

The general health of the mother, her previous obstetric history, and the availability of facilities for managing complications should all be taken into account when planning a pregnancy overseas. If antenatal care is carried out overseas but the mother plans to return to Britain for the delivery it must be remembered that most airlines do not allow passengers to travel after 35 weeks of pregnancy.

Live vaccinations are best not given during pregnancy though if someone unprotected against yellow fever is going to live in a high risk area the theoretical risk of vaccination is outweighed by the serious nature of the illness. If the vaccine is not given, a doctor's letter endorsed with a health board or authority stamp to say the inoculation is contraindicated is usually accepted. Inactivated poliomyelitis vaccine may be used instead of oral live vaccine.

A mother immunised against tetanus passes on protection to her baby over the neonatal period and a booster can be given during pregnancy if necessary. Hepatitis $A$ in pregnancy may be more severe and also result in premature labour. Prevention with normal immunoglobulin should be encouraged for those at risk.

Malarial prophylaxis should be maintained throughout pregnancy, as risks from all the drugs except Fansidar are less than the danger of malaria to the mother and fetus. Fansidar should not be taken during the last trimester or when breast feeding. There is a theoretical risk to the fetus from the antifolate drugs pyrimethamine and proguanil, although these drugs have been used for many years without serious problems being recognised in humans.

\section{Contraception}

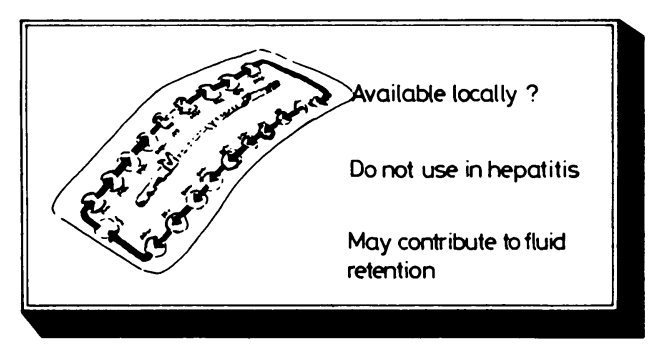

Those using oral contraceptives should be aware that absorption may be affected during gastrointestinal illnesses, that some brands may not be available locally, and that they may be continued over the usual break in the cycle if menstruation is going to occur at an inconvenient time such as during a long journey. They may contribute to the fluid retention that some people experience in hot climates, and they should not be taken by women suffering from hepatitis.

Dr Eric Walker, MRCP, MRCGP, and Dr Glyn Williams, MRCP, DTM\&H, are lecturers in the University Department of infectious diseases, Ruchill Hospital, Glasgow. 